PROCEEDINGS OF THE

AMERICAN MATHEMATICAL SOCIETY

Volume 127, Number 10, Pages 3021-3025

S 0002-9939(99)04837-6

Article electronically published on April 23, 1999

\title{
SURFACES WITH HARMONIC INVERSE MEAN CURVATURE IN SPACE FORMS
}

\author{
ATSUSHI FUJIOKA
}

(Communicated by Christopher Croke)

\begin{abstract}
We define surfaces with harmonic inverse mean curvature in space forms and generalize a theorem due to Lawson by which surfaces of constant mean curvature in one space form isometrically correspond to those in another. We also obtain an immersion formula, which gives a deformation family for these surfaces.
\end{abstract}

\section{INTRODUCTION}

In classical differential geometry, surfaces of constant mean curvature have been studied for a long time. As a generalization of surfaces of constant mean curvature in $\mathbf{R}^{3}$, Bobenko introduced surfaces with harmonic inverse mean curvature and obtained an immersion formula for these surfaces ([B2]).

In this paper we shall define surfaces with harmonic inverse mean curvature in space forms and generalize a theorem due to Lawson by which surfaces of constant mean curvature in one space form isometrically correspond to those in another ([L], $[\mathrm{P}],[\mathrm{F}])$. We shall also obtain an immersion formula, which gives a deformation family for these surfaces.

\section{§1. Definition of Surfaces With harmonic inverse mean CuRvature}

For $c=0, \pm 1$ we define a 1-dimensional Riemannian manifold $I_{c}$ by

$$
I_{c}=\left\{\begin{array}{ll}
\left(\mathbf{R}, g_{c}\right) & \text { if } c=0,1, \\
\left((-1,1), g_{c}\right) & \text { if } c=-1,
\end{array} \quad \text { where } \quad g_{c}=\frac{1}{\left(1+c t^{2}\right)^{2}} d t^{2} .\right.
$$

Let $M$ be a Riemann surface and $z$ a local holomorphic coordinate on $M$. Then we have the following:

Proposition 1.1. $\varphi: M \rightarrow I_{c}$ is harmonic if and only if $\varphi$ satisfies the following partial differential equation:

$$
\frac{\partial^{2} \varphi}{\partial z \partial \bar{z}}-\frac{2 c \varphi}{1+c \varphi^{2}}\left|\frac{\partial \varphi}{\partial z}\right|^{2}=0
$$

Received by the editors March 20, 1997 and, in revised form, December 4, 1997.

1991 Mathematics Subject Classification. Primary 53A10; Secondary 53A05.

Key words and phrases. Constant mean curvature surfaces, surfaces with prescribed mean curvature.

(C)1999 American Mathematical Society 
Equation (1.1) can be directly solved:

$$
\begin{cases}\varphi=\frac{\sqrt{-1}(h-\bar{h})}{|h|^{2}} & c=0, \\ \varphi=\frac{\sqrt{-1}(h-\bar{h})}{|h|^{2}-c} \text { or } \frac{|h|^{2}-c}{\sqrt{-1}(h-\bar{h})} & c= \pm 1,\end{cases}
$$

where $h: M \rightarrow \mathbf{C}$ is a holomorphic function such that $|h|^{2}-c \neq 0$ or $\operatorname{Im} h \neq 0$ on $M$. In the following we assume that $\operatorname{Im} h \neq 0,|h|^{2}-c \neq 0$ for $c=0, \pm 1$ on $M$. Let $\mathfrak{M}^{3}(c)$ be the simply connected 3-dimensional space form of curvature $c$.

Definition. A conformal immersion $F: M \rightarrow \mathfrak{M}^{3}(c)$ is called a surface with harmonic inverse mean curvature in $\mathfrak{M}^{3}(c)$ if the inverse of its mean curvature satisfies the equation (1.2).

Remark. If we restrict to the case $c=0$, this definition is given by Bobenko [B2]. For examples of surfaces with harmonic inverse mean curvature in $\mathfrak{M}^{3}(0)=\mathbf{R}^{3}$, see $[\mathrm{B} 2],[\mathrm{CK}]$.

\section{§2. Differential EQuations of SURFaces}

We consider $\mathbf{R}^{4}$ with the scalar product given by

$$
\langle a, b\rangle_{c}=c a_{0} b_{0}+\sum_{k=1}^{3} a_{k} b_{k},
$$

where

$$
a=\left(\begin{array}{c}
a_{0} \\
a_{1} \\
a_{2} \\
a_{3}
\end{array}\right), \quad b=\left(\begin{array}{c}
b_{0} \\
b_{1} \\
b_{2} \\
b_{3}
\end{array}\right) \in \mathbf{R}^{4} .
$$

Then $\mathfrak{M}^{3}(c)$ is embedded in $\mathbf{R}^{4}$ by

$$
\begin{aligned}
& \mathfrak{M}^{3}(0)=\left\{x \in \mathbf{R}^{4} ; x_{0}=0\right\}, \\
& \mathfrak{M}^{3}(1)=\left\{x \in \mathbf{R}^{4} ;\langle x, x\rangle_{1}=1\right\}, \\
& \mathfrak{M}^{3}(-1)=\text { a connected component of }\left\{x \in \mathbf{R}^{4} ;\langle x, x\rangle_{-1}=-1\right\} .
\end{aligned}
$$

Let $F: M \rightarrow \mathfrak{M}^{3}(c)$ be a conformal immersion, let $e^{u} d z d \bar{z}$ be the induced metric on $M$, and let $N$ be the unit normal to $M$. Direct computation shows that the Gauss-Codazzi equations have the following form (see [B1]):

$$
\left\{\begin{array}{l}
u_{z \bar{z}}+\frac{1}{2}\left(H^{2}+c\right) e^{u}-2|Q|^{2} e^{-u}=0, \\
Q_{\bar{z}}=\frac{1}{2} H_{z} e^{u},
\end{array}\right.
$$

where $Q=\left\langle F_{z z}, N\right\rangle_{c},\left\langle F_{z \bar{z}}, N\right\rangle_{c}=\frac{1}{2} H e^{u}$.

In the following we assume that $M$ is simply connected. We put

$$
\mathcal{C}_{H}=\left\{F: M \rightarrow \mathfrak{M}^{3}(c) ; F\right. \text { is a conformal immersion }
$$

$$
\text { with mean curvature } H\} / \operatorname{Iso}_{0}\left(\mathfrak{M}^{3}(c)\right) \text {, }
$$

where $\operatorname{Iso}_{0}\left(\mathfrak{M}^{3}(c)\right)$ is the identity component of the isometry group of $\mathfrak{M}^{3}(c)$. Then we have

$$
\mathcal{C}_{H} \cong\left\{(u, Q) ;(u, Q) \text { is a solution of }(\mathrm{GC})_{c}\right\}
$$


§3. An immersion formula: The CASE $H=\frac{|h|^{2}-c}{\sqrt{-1}(h-h)}$

In this section we put $H=H_{c}=\frac{|h|^{2}-c}{\sqrt{-1}(h-h)}$.

\section{Theorem 3.1.}

$$
\mathcal{C}_{H_{0}} \cong \mathcal{C}_{H_{1}} \cong \mathcal{C}_{H_{-1}}
$$

Proof. Let $(u, Q)$ be a solution of $(\mathrm{GC})_{c}$ for $c= \pm 1$. We put

$$
e^{u^{\prime}}=\left|\frac{h^{2}-c}{h^{2}}\right|^{2} e^{u}, \quad Q^{\prime}=\frac{h^{2}-c}{h^{2}} Q .
$$

Then it is easy to see that $\left(u^{\prime}, Q^{\prime}\right)$ is a solution of $(\mathrm{GC})_{0}$. Conversely, we can easily construct a solution of $(\mathrm{GC})_{c}$ for $c= \pm 1$ from one of $(\mathrm{GC})_{0}$.

Remark. Theorem 3.1 is considered as a generalization of a theorem due to Lawson by which surfaces of constant mean curvature in one space form isometrically correspond to those in another $([\mathrm{L}],[\mathrm{P}],[\mathrm{F}])$.

Let $(u, Q)$ be a solution of $(\mathrm{GC})_{0}$. For a $2 \times 2$ matrix-valued function $\Phi$ on $M$, we consider the following equations:

$$
\left\{\begin{array}{l}
\Phi_{z}=U \Phi, \\
\Phi_{\bar{z}}=V \Phi
\end{array}\right.
$$

where

$$
\begin{gathered}
U=\left(\begin{array}{cc}
\frac{1}{2} u_{z} & -Q e^{-\frac{1}{2} u} \\
\frac{1}{2} \lambda H_{0} e^{\frac{1}{2} u} & 0
\end{array}\right), \quad V=\left(\begin{array}{cc}
0 & -\frac{1}{2} \lambda^{-1} H_{0} e^{\frac{1}{2} u} \\
\bar{Q} e^{-\frac{1}{2} u} & \frac{1}{2} u_{\bar{z}}
\end{array}\right), \\
\lambda=\left(1+\frac{2}{\bar{h}} \mu\right) /\left(1+\frac{2}{h} \mu\right), \quad \mu \in \mathbf{C} .
\end{gathered}
$$

Note that the integrability conditions of (3.1),

$$
U_{\bar{z}}-V_{z}+[U, V]=0,
$$

are equivalent to $(\mathrm{GC})_{0}$. We put

$$
E=\left(\begin{array}{ll}
1 & 0 \\
0 & 1
\end{array}\right), \sigma_{1}=\left(\begin{array}{ll}
0 & 1 \\
1 & 0
\end{array}\right), \sigma_{2}=\left(\begin{array}{cc}
0 & -\sqrt{-1} \\
\sqrt{-1} & 0
\end{array}\right), \sigma_{3}=\left(\begin{array}{cc}
1 & 0 \\
0 & -1
\end{array}\right)
$$

and use the following identification according to $c$ :

$$
\begin{gathered}
x=-\sqrt{-1} \sum_{k=1}^{3} x_{k} \sigma_{k} \longleftrightarrow x=\left(\begin{array}{c}
0 \\
x_{1} \\
x_{2} \\
x_{3}
\end{array}\right) \in \mathbf{R}^{4} \quad \text { if } c=0, \\
x=x_{0} E+\sqrt{-1} \sum_{k=1}^{3} x_{k} \sigma_{k} \longleftrightarrow x=\left(\begin{array}{l}
x_{0} \\
x_{1} \\
x_{2} \\
x_{3}
\end{array}\right) \in \mathbf{R}^{4} \quad \text { if } c=1,
\end{gathered}
$$




$$
x=x_{0} E+\sum_{k=1}^{3} x_{k} \sigma_{k} \longleftrightarrow x=\left(\begin{array}{c}
x_{0} \\
x_{1} \\
x_{2} \\
x_{3}
\end{array}\right) \in \mathbf{R}^{4} \quad \text { if } c=-1 .
$$

Similar computation as in [B1] gives the following:

Theorem 3.2. (i) (Bobenko [B2]) Let $\Phi=\Phi(z, \bar{z}, \mu), \mu \in \mathbf{R}$, be a $\mathbf{R}_{+} S U(2)$-valued solution of (3.1) such that $\operatorname{det} \Phi$ is independent of $\mu$. Then $\Phi^{-1} \frac{\partial}{\partial \mu} \Phi$ is a surface with harmonic inverse mean curvature in $\mathfrak{M}^{3}(0)$.

(ii) Let $\Phi_{1}=\Phi\left(z, \bar{z}, \mu_{1}\right), \Phi_{2}=\Phi\left(z, \bar{z}, \mu_{2}\right), \mu_{1}, \mu_{2} \in \mathbf{R}$, with $\mu_{1} \neq \mu_{2}$ be $\mathbf{R}_{+} S U(2)$-valued solutions of (3.1) such that $\operatorname{det} \Phi_{1}=\operatorname{det} \Phi_{2} . \quad$ Then $\Phi_{1}^{-1} \Phi_{2}$ is a surface with harmonic inverse mean curvature in $\mathfrak{M}^{3}(1)$.

(iii) Let $\Phi=\Phi(z, \bar{z}, \mu), \mu \in \sqrt{-1} \mathbf{R}$, be a solution of (3.1) such that $\operatorname{det} \Phi \in$ $\mathbf{R} \backslash\{0\}$. Then $\Phi^{-1} \sigma_{2} \bar{\Phi} \sigma_{2}$ is a surface with harmonic inverse mean curvature in $\mathfrak{M}^{3}(-1)$.

Remark. (i) In Theorem $3.2 \mu$ plays a role of deformation parameter for surfaces with harmonic inverse mean curvature.

(ii) If we replace $\mathfrak{M}^{3}(c)$ with the 3 -dimensional pseudo-Riemannian space form of curvature $c$, we can define spacelike surfaces with harmonic inverse mean curvature. Then similar arguments as above can be applied to these surfaces.

$$
\text { §4. An immersion formula: The CASE } H=\frac{\sqrt{-1}(h-\bar{h})}{|h|^{2}-c}, c= \pm 1
$$

In the following we put $H=\frac{\sqrt{-1}(h-\bar{h})}{|h|^{2}-c}, c= \pm 1$. As in the previous section, we consider the following equations:

$$
\left\{\begin{array}{l}
\Phi_{z}=U \Phi \\
\Phi_{\bar{z}}=V \Phi
\end{array}\right.
$$

where

$$
\begin{gathered}
U=\left(\begin{array}{cc}
\frac{1}{4} u_{z} & -Q e^{-\frac{1}{2} u} \\
\frac{1}{2} \lambda\left(H_{\alpha}-c\right) e^{\frac{1}{2} u} & -\frac{1}{4} u_{z}
\end{array}\right), \\
V=\left(\begin{array}{cc}
-\frac{1}{4} u_{\bar{z}} & -\frac{1}{2} \bar{\lambda}\left(H_{\alpha}+c\right) e^{\frac{1}{2} u} \\
\bar{Q} e^{-\frac{1}{2} u} & \frac{1}{4} u_{\bar{z}}
\end{array}\right), \\
\lambda=\frac{1}{\bar{\alpha}} \frac{h^{2}-c}{\alpha^{2} h^{2}-c}, \quad H_{\alpha}=\frac{\sqrt{-1}(\alpha h-\bar{\alpha} \bar{h})}{|h|^{2}-c}, \\
\alpha \in\{\mu \in \mathbf{C} ;|\mu|=1\} .
\end{gathered}
$$

Note that the integrability conditions of (4.1),

$$
U_{\bar{z}}-V_{z}+[U, V]=0,
$$

are equivalent to $(\mathrm{GC})_{c}$. Using (4.1), we can obtain an immersion formula in a similar manner to that of Theorem 3.2. 


\section{ACKNOWLEDGEMENTS}

The author would like to thank Professor Takushiro Ochiai for his useful suggestions and constant encouragement, and also Hideko Hashiguchi and Masanori Higaki. Jun-ichi Inoguchi and the referee pointed out that there was an inaccuracy in formulas (1.1) and (1.2). The author would also like to express his sincere gratitude to both of them.

\section{REFERENCES}

[B1] A. I. Bobenko, Constant mean curvature surfaces and integrable equations, Russian Math. Surveys 46:4 (1991), 1-45. MR 93b:53009

[B2] A. I. Bobenko, Surfaces in terms of 2 by 2 matrices. Old and new integrable cases, Harmonic maps and Integrable Systems (A.Fordy and J.C.Wood, eds.), Aspects of Mathematics, Vieweg, 1994, pp. 83-127. CMP 94:09

[CK] G. Colares and K. Kenmotsu, Isometric deformation of surfaces in $\mathbf{R}^{3}$ preserving the mean curvature function, Pacific J. Math. 136 (1989), 71-80. MR 89m:53011

[F] A. Fujioka, Harmonic maps and associated maps from simply connected Riemann surfaces into the 3-dimensional space forms, Tôhoku Math. J. 47 (1995), 431-439. MR 96h:58045

[L] B. Lawson, Complete minimal surfaces in $S^{3}$, Ann. of Math. 92 (1970), 335-374. MR 42:5170

[P] B. Palmer, Spacelike constant mean curvature surfaces in pseudo-Riemannian space forms, Ann. Global Anal. Geom. 8 (1990), 217-226. MR 91m:53051

Department of Mathematics, Faculty of Science, Kanazawa University, KakumaMACHI, KANAZAWA, 920-1192 JAPAN

E-mail address: fujioka@kappa.s.kanazawa-u.ac.jp

Current address: Graduate School of Natural Science and Technology, Kanazawa University, Kakuma-machi, Kanazawa, 920-1192 Japan 